\title{
Preoperative plus postoperative neutrophil-lymphocyte ratio for predicting overall survival following partial hepatectomy for hepatocellular carcinoma
}

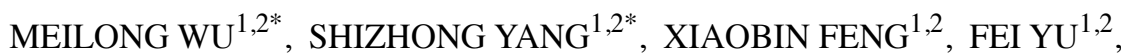 \\ XIANGCHEN LIU ${ }^{1,2}$ and JIAHONG DONG ${ }^{1,2}$ \\ ${ }^{1}$ School of Clinical Medicine, Tsinghua University, Haidian, Beijing 100084; \\ ${ }^{2}$ Hepatopancreatobiliary Center, Beijing Tsinghua Changgung Hospital, School of Clinical Medicine, \\ Tsinghua University, Changping, Beijing 102218, P.R. China
}

Received April 25, 2020; Accepted September 11, 2020

DOI: $10.3892 / \mathrm{ol} .2020 .12238$

\begin{abstract}
The preoperative neutrophil-lymphocyte ratio (NLR) and the postoperative NLR have been reported to be prognostic factors for malignant tumors. However, the prognostic value of combining the preoperative NLR and postoperative NLR for hepatocellular carcinoma (HCC) remains unclear. In the present study, a cohort of 70 patients with primary HCC were retrospectively reviewed. The optimal cut-offs for continuous variables were determined by the maximally selected rank statistics. The prognostic factors included preoperative NLR, postoperative NLR, preoperative NLR plus postoperative NLR, change in postoperative NLR, and postoperative NLR minus preoperative NLR. The predictive powers of the aforementioned prognostic factors were analyzed by the area under the time-dependent receiver operating characteristic (td-AUC) curve. Prognostic values were assessed by univariate and multivariate analyses. An increased preoperative NLR was found to be associated with higher preoperative neutrophil levels, lower preoperative lymphocyte levels and larger tumor sizes (all $\mathrm{P}<0.05)$. An increased postoperative NLR was associated with higher postoperative neutrophil levels and lower postoperative lymphocyte levels (all $\mathrm{P}<0.05$ ). Multivariate analysis identified the preoperative NLR plus postoperative NLR as an independent prognostic risk factor (HR, 2.985; 95\% CI, 1.648-5.407; $\mathrm{P}<0.001$ ). The preoperative NLR plus postoperative NLR had higher td-AUC values than the preoperative NLR, postoperative NLR, postoperative NLR
\end{abstract}

Correspondence to: Professor Jiahong Dong, School of Clinical Medicine, Tsinghua University, 1 Tsinghua Yuan, Haidian, Beijing 100084, P.R. China

E-mail: dongjiahong@mail.tsinghua.edu.cn

${ }^{*}$ Contributed equally

Key words: liver resection, neutrophil-lymphocyte ratio, preoperative, postoperative, hepatocellular carcinoma change, and postoperative NLR minus the preoperative NLR in the first to fourth years after surgery. The preoperative NLR plus postoperative NLR, considering both the preoperative and postoperative treatment phases, is a novel and promising prognostic factor for patients with $\mathrm{HCC}$ and requires further investigation in the future.

\section{Introduction}

Hepatocellular carcinoma (HCC) is an international health problem that places a substantial burden on the healthcare system (1). Low-cost treatment options are recommended for economic reasons. Recent studies have demonstrated that an increased preoperative neutrophil-lymphocyte ratio (NLR) is an economical independent prognostic indicator in several different types of malignancy, including $\operatorname{HCC}(2,3)$, glioblastoma multiforme (4), pancreatic neuroendocrine tumors (5), gastric (6), ovarian (7), renal (8) and breast cancer (9).

Persistent inflammation promotes and accelerates the progression of malignant tumors at different stages of tumor development, including initiation, progression, malignant conversion, invasion and metastasis (10). In recent years, certain studies have focused on the effect of postoperative NLR on the prognosis of patients with malignant tumors (11-21) and have reported that an increased postoperative NLR was associated with poorer survival outcomes in patients with solid tumors $(12,15,21)$. Certain studies have begun to focus on the association between preoperative NLR and postoperative NLR: Postoperative NLR changes for advanced tumors $(13,14)$; the preoperative plus the postoperative NLR for gastric cancer (15); and the postoperative NLR minus preoperative NLR for HCC $(3,16)$, colorectal (17), non-small-cell lung (18) and gastric cancer $(19,20)$. Some of these studies were based on the hypothesis that the postoperative NLR is higher than the preoperative NLR following treatment, which may be due to a protumor inflammatory response, and they found that patients with a postoperative NLR higher than the preoperative NLR had a poorer prognosis $(3,13,14,16-20)$. However, the association between the preoperative NLR and postoperative NLR remains controversial, and it may not be appropriate to 
study the postoperative NLR in the context of the preoperative NLR. Peng et al (16) reported that the balance between the immune response and the inflammatory response may change following the surgical removal of HCC lesions.

$\mathrm{HCC}$ is a typical example of an inflammation-related tumor, as $>90 \%$ of HCC cases arise in the context of hepatic injury and inflammation $(1,22)$. The prognostic utility of the preoperative NLR and postoperative NLR for HCC requires further study. Although preoperative NLR minus postoperative NLR was identified as an independent prognostic factor for HCC in previous studies, no significant differences were identified between the two groups for commonly evaluated clinicopathological features $(3,16)$. Therefore, the application and reliability of the postoperative NLR minus preoperative NLR and other associated combinations for the prognosis of $\mathrm{HCC}$ require further study.

\section{Materials and methods}

Patient selection. A total of 70 patients undergoing partial hepatectomy for primary HCC admitted to Beijing Tsinghua Chang Gung Hospital affiliated with Tsinghua University (Beijing, China) between December 2014 and July 2019 were retrospectively analyzed. The mean patient age was $56.97 \pm 10.06$ years (range, 33-81 years). The HCC cohort consisted of 54 males (77.1\%) and 16 females (22.9\%). The study was approved by the Ethics Committee of Beijing Tsinghua Chang Gung Hospital (Beijing, China). The inclusion criteria were as follows: i) pathological diagnosis of primary $\mathrm{HCC}$ with a planned treatment of curative resection; ii) no redness or exudation from the incision when the patient was discharged from the hospital and no infections or complications at the time of discharge; iii) complete demographic data; iv) complete laboratory and imaging examinations; and v) regular follow-up following the operation.

Clinicopathological variables. All patient data (including laboratory indicators, imaging data and pathology data) were obtained from the hospital's electronic information system. The preoperative laboratory indicators were taken from the first laboratory results after admission. The postoperative laboratory examinations were performed 1-2 days before discharge after all complications had been resolved. At that time, the surgical incisions had no redness or exudations. The overall survival (OS) time was defined as the day of surgery until the day the patient died or the last follow-up day. All the deceased patients died as a result of their tumors.

The study variables included the following: (1) general information (age and sex); (2) blood test results [white blood cells, neutrophils, lymphocytes, hepatitis B surface antigen (HBsAg), albumin, and alpha-fetoprotein (AFP)]; (3) pathological indicators (cirrhosis, tumor number, tumor size and tumor differentiation); (4) prognostic information (patient survival time); and (5) complications. According to the World Health Organization standards, the degree of differentiation of HCC was divided into poorly-, moderately- and highly-differentiated (23).

The present study investigated the effect of the preoperative NLR, postoperative NLR, postoperative NLR change, preoperative NLR plus postoperative NLR and postoperative NLR minus preoperative NLR on the prognosis of patients with HCC following resection. The NLR was defined as the ratio of the absolute number of neutrophils to the absolute number of lymphocytes (19). The postoperative change was defined as the postoperative NLR divided by the preoperative NLR (13). The postoperative NLR minus preoperative NLR was defined as the postoperative NLR value minus the preoperative NLR value $(3,18)$. The preoperative NLR plus the postoperative NLR value was calculated based on the preoperative NLR and postoperative NLR cut-off values as follows: Patients with both an increased preoperative NLR and postoperative NLR were assigned a score of 2, patients showing either an increased preoperative NLR or an elevated postoperative NLR were assigned a score of 1 , and patients in whom neither the preoperative NLR nor the postoperative NLR was increased were assigned a score of 0 .

Statistical analysis. Categorical data are reported as the number and percentage of cases. The chi-square test or Fisher's exact test were used to analyze the categorical data. Continuous variables are reported as the mean \pm standard deviation and were compared using Student's t-test or analysis of variance $(3,24)$. A Cox proportional hazard model was used for the univariate and multivariate analyses. The survival curve was drawn with the 'Surv_fit' function in the R software (25).

The optimal cut-off for continuous variables was determined by using the maximally selected rank statistics by the 'surv_cutpoint' function of the 'survminer' R package $(26,27)$. The time-dependent receiver operating characteristic (td-ROC) curve was estimated to assess the performance of the preoperative NLR, postoperative NLR, postoperative NLR change, preoperative NLR plus postoperative NLR, and postoperative NLR minus preoperative NLR. The time-dependent area under the curve (td-AUC) was calculated by the survival ROC function of the R software, and a larger td-AUC indicated more accurate prognostic stratification (25). All data were analyzed using SPSS 26.0 software (IBM Corp.) and $\mathrm{R}$ version 3.6.1 (http://www.r-project. org/). Graphs were created using $\mathrm{R}$ software. $\mathrm{P}<0.05$ was considered to indicate a statistically significant difference.

\section{Results}

Baseline characteristics. A total of 70 patients with $\mathrm{HCC}$ were included in the present study. Hepatitis B was diagnosed in 59 patients. The mean tumor size was $5.67 \pm 3.92 \mathrm{~cm}$, and the number of patients with solitary tumors was 57 (81.4\%). The number of patients with cirrhosis was 33 (47.1\%). Complications included biliary leakage $(n=2,2.86 \%)$, pleural effusion $(n=56$, $80 \%)$, pneumonia $(n=3,4.29 \%)$ and perihepatic effusion $(n=1$, $1.43 \%$ ). The mean preoperative white blood cell, neutrophil and lymphocyte counts and the postoperative white blood cell, neutrophil and lymphocyte counts were 5.41 $\pm 1.91,3.26 \pm 1.76$, $1.55 \pm 0.56,5.98 \pm 2.14 \mathrm{ng} / \mathrm{ml}, 3.82 \pm 1.61$ and $1.39 \pm 0.65 \mathrm{mg} / 1$, respectively.

Optimal cut-offs for continuous variables. The optimal cut-offs of the preoperative NLR, postoperative NLR, postoperative NLR change and postoperative NLR minus preoperative NLR were $3.46,4.33,-0.25$ and 3.42 , respectively. 
Table I. Comparison of baseline clinical characteristics according to preoperative NLR and postoperative NLR values.

\begin{tabular}{|c|c|c|c|c|c|c|c|}
\hline Baseline variable & $\begin{array}{l}\text { Overall, } \\
(\mathrm{n}=70)\end{array}$ & $\begin{array}{c}\text { Preoperative } \\
\text { NLR } \leq 3.46, \\
(\mathrm{n}=61)\end{array}$ & $\begin{array}{l}\text { Preoperative } \\
\text { NLR }>3.4 \\
\quad(n=9)\end{array}$ & P-value & $\begin{array}{l}\text { Postoperative } \\
\text { NLR } \leq 4.33 \\
(\mathrm{n}=58)\end{array}$ & $\begin{array}{l}\text { Postoperative } \\
\text { NLR }>4.33 \\
\quad(n=12)\end{array}$ & P-value \\
\hline Age, years & $56.97 \pm 10.06$ & $57.33 \pm 9.84$ & $54.56 \pm 11.81$ & 0.444 & $56.57 \pm 10.16$ & $58.92 \pm 9.75$ & 0.466 \\
\hline Sex, male & $54(77.1)$ & $45(73.8)$ & $9(100)$ & 0.082 & $42(72.3)$ & $12(100)$ & 0.055 \\
\hline HBsAg (+) & $59(84.3)$ & $51(83.6)$ & $8(88.9)$ & 1.000 & $49(84.5)$ & $10(83.3)$ & 1.000 \\
\hline $\operatorname{AFP}(\mathrm{ng} / \mathrm{ml})$ & $662.48 \pm 808.76$ & $666.38 \pm 808.31$ & $636.06 \pm 860.53$ & 0.917 & $656.96 \pm 787.73$ & $689.16 \pm 941.62$ & 0.901 \\
\hline Preoperative WBC $\left(10^{9} / 1\right)$ & $5.41 \pm 1.91$ & $5.02 \pm 1.20$ & $8.10 \pm 3.40$ & $<0.001$ & $5.33 \pm 1.84$ & $5.84 \pm 2.28$ & 0.407 \\
\hline $\begin{array}{l}\text { Preoperative } \\
\text { neutrophil }\left(10^{9} / 1\right)\end{array}$ & $3.26 \pm 1.76$ & $2.81 \pm 0.87$ & $6.31 \pm 3.01$ & 0.001 & $1.68 \pm 0.22$ & $3.87 \pm 2.09$ & 0.187 \\
\hline $\begin{array}{l}\text { Preoperative } \\
\text { lymphocyte }\left(10^{9} / 1\right)\end{array}$ & $1.54 \pm 0.57$ & $1.63 \pm 0.56$ & $1.03 \pm 0.33$ & $<0.003$ & $1.61 \pm 0.57$ & $1.27 \pm 0.51$ & 0.069 \\
\hline Albumin (g/dl) & $42.53 \pm 8.53$ & $43.25 \pm 8.84$ & $37.68 \pm 3.30$ & 0.067 & $43.15 \pm 9.14$ & $39.55 \pm 3.41$ & 0.185 \\
\hline Postoperative WBC $\left(10^{9} / 1\right)$ & $5.98 \pm 2.14$ & $5.85 \pm 1.92$ & $6.86 \pm 3.27$ & 0.190 & $5.69 \pm 2.07$ & $7.42 \pm 1.94$ & 0.010 \\
\hline $\begin{array}{l}\text { Postoperative } \\
\text { neutrophil }\left(10^{9} / 1\right)\end{array}$ & $3.83 \pm 1.61$ & $3.70 \pm 1.50$ & $4.70 \pm 2.12$ & 0.080 & $3.47 \pm 1.39$ & $5.56 \pm 1.56$ & $<0.001$ \\
\hline $\begin{array}{l}\text { Postoperative } \\
\text { lymphocyte }\left(10^{9} / 1\right)\end{array}$ & $1.39 \pm 0.65$ & $1.41 \pm 0.56$ & $1.29 \pm 1.13$ & 0.627 & $1.49 \pm 0.66$ & $0.92 \pm 0.31$ & 0.005 \\
\hline Cirrhosis, yes & $33(47.1)$ & $28(45.9)$ & $5(55.6)$ & 0.854 & $27(46.6)$ & $6(50.0)$ & 0.828 \\
\hline Tumor size, $\mathrm{cm}$ & $5.67 \pm 3.92$ & $5.04 \pm 3.51$ & $9.88 \pm 4.19$ & 0.001 & $5.46 \pm 3.74$ & $6.68 \pm 4.77$ & 0.327 \\
\hline Tumor number, multiple & $13(18.57)$ & $10(16.39)$ & $2(22.22)$ & 1.000 & $10(17.24)$ & $2(16.67)$ & 1.000 \\
\hline Differentiation, poor & $8(11.43)$ & $6(9.84)$ & $2(22.22)$ & 0.597 & $5(8.62)$ & $3(25)$ & 0.261 \\
\hline Biliary leakage & $2(2.86)$ & $2(3.28)$ & $0(0)$ & 1.000 & $2(3.45)$ & $0(0)$ & 1.000 \\
\hline Pleural effusion & $56(70)$ & $49(80.33)$ & $7(77.78)$ & 1.000 & 45 (77.59) & $12(91.67)$ & 0.267 \\
\hline Pneumonia & $3(4.28)$ & $3(4.91)$ & $0(0)$ & 0.858 & $3(5.17)$ & $0(0)$ & 0.982 \\
\hline Perihepatic effusion & $1(1.43)$ & $1(1.66)$ & $0(0)$ & 1.000 & $1(1.72)$ & $0(0)$ & 1.000 \\
\hline
\end{tabular}

Data are presented as mean \pm standard deviation or $n(\%)$. AFP, alpha-fetoprotein; HBsAg, hepatitis B surface antigen; NLR, neutrophil-lymphocyte ratio; WBC, white blood cells.

The optimal cut-off for age and tumor size were also calculated (47-years-old and $5 \mathrm{~cm}$, respectively).

Clinical and pathological data for preoperative NLR and postoperative NLR. An increased preoperative NLR was associated with a higher preoperative white blood cell count $(\mathrm{P}<0.001)$, higher preoperative neutrophil count $(\mathrm{P}=0.001)$, lower preoperative lymphocyte count $(\mathrm{P}<0.003)$ and larger tumor size $(\mathrm{P}=0.001)$. An increased postoperative NLR was associated with a higher postoperative white blood cell count $(\mathrm{P}=0.010)$, higher postoperative neutrophil count $(\mathrm{P}<0.001)$, and lower postoperative lymphocyte count $(\mathrm{P}=0.005)$. There was no correlation between an increased preoperative NLR and the occurrence of complications. Similarly, there was no statistically significant difference in complications between the low and high postoperative NLR groups (Table I).

Survival analysis. A total of 19 patients (27.14\%) died during the follow-up period. The mean survival duration was 12 months (range, 2-52 months). The 1-, 2-, 3- and 4-year OS rates were $87.1,74.0,72.8$ and $72.8 \%$, respectively.

The 70 patients with HCC were divided into 2 groups according to their preoperative NLR values: preoperative NLR $\leq 3.46(n=61)$ and preoperative NLR>3.46 $(n=9$; Fig. 1A).
Likewise, the patients with HCC were divided into 2 groups according to their postoperative NLR profiles: postoperative NLR $\leq 4.33(n=58)$ and postoperative NLR $>4.33(n=12$; Fig. 1B). Using the Surv_fit function to analyze patient survival, high preoperative NLR and high postoperative NLR were significantly associated with $\mathrm{OS}(\mathrm{P}=0.00068$ and $\mathrm{P}=0.0044$, respectively; Fig. $1 \mathrm{~A}$ and $\mathrm{B}$ ).

The 70 patients with HCC were divided into 2 groups according to their postoperative NLR change and postoperative NLR minus preoperative NLR values: postoperative NLR change $>-0.25(n=9)$ and postoperative NLR minus preoperative NLR $\geq 3.42(n=7)$. In the Surv_fit function analysis, a high postoperative NLR change was not a significant prognostic factor $(\mathrm{P}=0.27$; Fig. $1 \mathrm{C})$, but a high postoperative NLR minus preoperative NLR of $\geq 3.42$ was significantly associated with a decreased OS ( $\mathrm{P}=0.0048)$ (Fig. 1D).

Patients with preoperative NLR plus postoperative NLR scores of 0 had the most favorable outcomes, and patients with preoperative NLR plus postoperative NLR=2 had the poorest prognosis. These findings indicated that an increased preoperative NLR and an increased postoperative NLR were significantly associated with poor survival compared with a low preoperative NLR and/or postoperative NLR $(\mathrm{P}=0.00033$; Fig. 1E). 

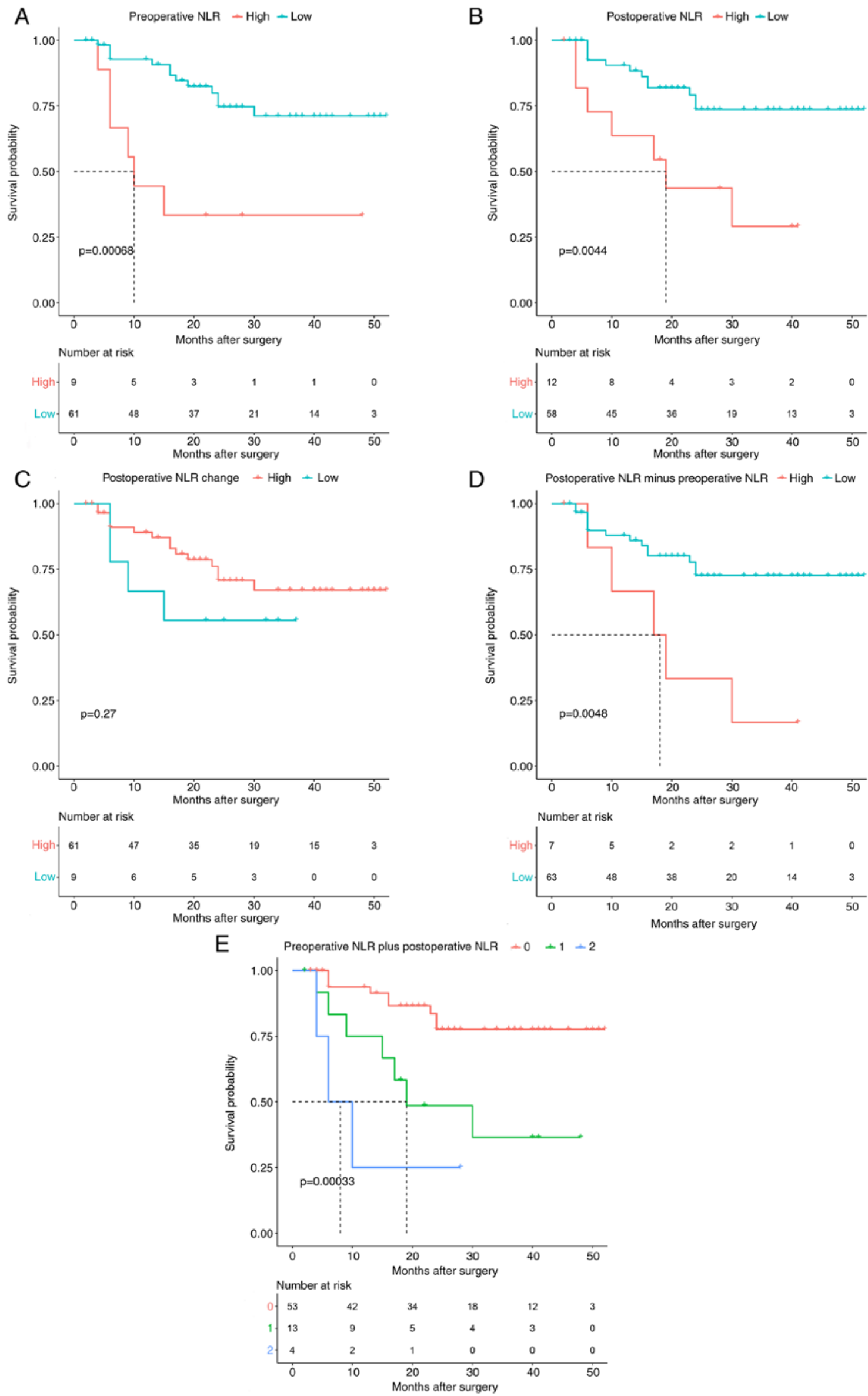

Figure 1. Kaplan-Meier survival curves for overall survival in 70 patients undergoing partial hepatectomy for primary hepatocellular carcinoma. (A) Preoperative NLR, (B) postoperative NLR, (C) postoperative NLR change, (D) postoperative NLR minus preoperative NLR, and (E) preoperative NLR plus postoperative NLR. NLR, neutrophil-lymphocyte ratio. 
Table II. Univariate and multivariate analyses of prognostic factors of overall survival for patients with hepatocellular carcinoma.

\begin{tabular}{|c|c|c|c|c|c|c|}
\hline \multirow[b]{2}{*}{ Baseline variable } & \multicolumn{3}{|c|}{ Univariate analyses } & \multicolumn{3}{|c|}{ Multivariate analyses } \\
\hline & HR & $95 \% \mathrm{CI}$ & P-value & HR & $95 \% \mathrm{CI}$ & P-value \\
\hline Age, $>47$ years & 0.696 & $0.250-1.936$ & 0.488 & & & \\
\hline Sex, male & 3.010 & $0.694-13.045$ & 0.141 & & & \\
\hline HBsAg (+) & 1.521 & $0.351-6.590$ & 0.575 & & & \\
\hline $\mathrm{AFP},>20 \mathrm{ng} / \mathrm{ml}$ & 2.340 & $0.681-8.047$ & 0.177 & & & \\
\hline Preoperative WBC, $10^{9} / 1$ & 1.030 & $0.830-1.297$ & 0.786 & & & \\
\hline Preoperative neutrophil, $10^{9} / 1$ & 1.087 & $0.855-1.334$ & 0.427 & & & \\
\hline Preoperative lymphocyte, $10^{9} / 1$ & 0.534 & $0.255-1.266$ & 0.154 & & & \\
\hline Albumin, g/dl & 0.920 & $0.841-1.007$ & 0.071 & & & \\
\hline Postoperative WBC, $10^{9} / 1$ & 1.040 & $0.848-1.277$ & 0.704 & & & \\
\hline Postoperative neutrophil, $10^{9} / 1$ & 1.224 & $0.939-1.596$ & 0.135 & & & \\
\hline Postoperative lymphocyte, $10^{9} / 1$ & 0.682 & $0.291-1.599$ & 0.379 & & & \\
\hline Cirrhosis, yes & 0.943 & $0.382-2.330$ & 0.899 & & & \\
\hline Tumor size, $>5 \mathrm{~cm}$ & 2.795 & $1.099-7.110$ & 0.031 & & & \\
\hline Tumor number, multiple & 1.549 & $0.513-4.681$ & 0.438 & & & \\
\hline Differentiation, poor & 3.079 & $1.001-9.473$ & 0.050 & & & \\
\hline Biliary leakage & 0.048 & $0.000-70634.465$ & 0.675 & & & \\
\hline Pleural effusion & 0.688 & $0.227-2.086$ & 0.508 & & & \\
\hline Pneumonia & 0.941 & $0.126-7.060$ & 0.953 & & & \\
\hline Perihepatic effusion & 0.048 & $0.000-103525.973$ & 0.683 & & & \\
\hline Preoperative NLR & 4.618 & $1.728-12.339$ & 0.002 & & & \\
\hline Postoperative NLR & 3.512 & $1.381-8.934$ & 0.008 & & & \\
\hline Preoperative NLR plus Postoperative NLR & 2.985 & $1.648-5.407$ & $<0.001$ & 2.985 & $1.648-5.407$ & $<0.001$ \\
\hline Postoperative NLR change & 0.548 & $0.182-1.654$ & 0.286 & & & \\
\hline Postoperative NLR minus Preoperative NLR & 3.296 & $1.181-9.199$ & 0.023 & & & \\
\hline
\end{tabular}

HR, hazard ratio; CI, confidence interval; AFP, alpha-fetoprotein; HBsAg, hepatitis B surface antigen; NLR, neutrophil-lymphocyte ratio; WBC, white blood cells.

Factors associated with prognosis. To identify the independent prognostic risk factors in patients with primary HCC undergoing partial hepatectomy, Cox univariate and multivariate analyses were performed. The results of the univariate analysis are shown in Table II. The univariate analysis showed that tumor size (HR, 2.795; 95\% CI, 1.099-7.110; $\mathrm{P}=0.031$ ), preoperative NLR plus postoperative NLR (HR, 2.985; 95\% CI, 1.648-5.407; $\mathrm{P}<0.001$ ), preoperative NLR (HR, 4.618; 95\% CI, 1.728-12.339; $\mathrm{P}=0.002)$, postoperative NLR (HR, 3.512; 95\% CI, 1.728-12.339; $\mathrm{P}=0.008)$, and postoperative NLR minus preoperative NLR (HR, 3.296; 95\% CI, 1.181-9.199; $\mathrm{P}=0.023$ ) were significantly associated with a poor OS. Subsequently, the multivariate analysis identified the preoperative NLR plus postoperative NLR (HR, 2.985; 95\% CI, 1.648-5.407; $\mathrm{P}<0.001$ ) as an independent prognostic risk factor (Table II).

Preoperative NLR plus postoperative NLR is superior to other factors in predicting prognosis. The td-ROC curves were used to assess the discriminatory abilities of the preoperative NLR plus postoperative NLR, preoperative NLR, postoperative NLR, postoperative NLR change, and postoperative NLR minus preoperative NLR in terms of prognosis. The preoperative NLR plus postoperative NLR had higher td-AUC values at 1 year $(0.76), 2$ years $(0.655), 3$ years $(0.685)$ and 4 years $(0.685)$ than the preoperative NLR ( 1 year, $\mathrm{td}-\mathrm{AUC}=0.733 ; 2$ years, $\mathrm{td}-\mathrm{AUC}=0.609 ; 3$ years, td-AUC=0.595; and 4 years, td-AUC=0.595; Fig. 2).

Compared with the postoperative NLR, the preoperative NLR plus postoperative NLR had higher td-AUC values ( 1 year, 0.76 vs. $0.657 ; 2$ years, 0.655 vs. $0.599 ; 3$ years, 0.685 vs. 0.641 ; and 4 years, 0.685 vs. 0.641 ; Fig. 2 ).

The preoperative NLR plus postoperative NLR had higher td-AUC values at 1 year $(0.76), 2$ years $(0.655), 3$ years $(0.685)$ and 4 years $(0.685)$ than the CNLR $(1$ year, td-AUC $=0.606$; 2 years, $\mathrm{td}-\mathrm{AUC}=0.54 ; 3$ years, $\mathrm{td}-\mathrm{AUC}=0.529$; and 4 years, td-AUC=0.529; Fig. 2). Similarly, the preoperative NLR plus postoperative NLR had a higher td-AUC than the postoperative NLR minus preoperative NLR in the first to fourth years (Fig. 2).

Clinical and pathological data for preoperative NLR plus postoperative NLR. Among the 70 patients, 53 (75.71\%) were classified as having a preoperative NLR plus postoperative NLR score of 0 , whereas $13(15.87 \%)$ and $4(0.06 \%)$ were 
Table III. Comparison of clinical and pathological data according to preoperative NLR plus postoperative NLR values.

\begin{tabular}{|c|c|c|c|c|}
\hline Baseline variable & $\begin{array}{c}\text { Preoperative NLR } \\
\text { plus postoperative } \\
\text { NLR }=0, n=53\end{array}$ & $\begin{array}{c}\text { Preoperative NLR } \\
\text { plus postoperative } \\
\text { NLR }=1, n=13\end{array}$ & $\begin{array}{l}\text { Preoperative NLR } \\
\text { plus postoperative } \\
\quad \text { NLR }=2, n=4\end{array}$ & P-value \\
\hline Age, years & $59.19 \pm 9.837$ & $55.08 \pm 11.507$ & $60.25 \pm 9.467$ & 0.641 \\
\hline Sex, male & $37(69.8)$ & $13(100)$ & $4(100)$ & 0.036 \\
\hline $\mathrm{HBsAg}(+)$ & $44(83)$ & $12(92.3)$ & $3(75)$ & 0.623 \\
\hline $\mathrm{AFP}, \mathrm{ng} / \mathrm{ml}$ & $683.522 \pm 801.952$ & $484.569 \pm 776.219$ & $961.883 \pm 1105.531$ & 0.552 \\
\hline Preoperative WBC, 109/1 & $5.018 \pm 1.195$ & $6.046 \pm 3.040$ & $7.458 \pm 3.154$ & $0.004^{\mathrm{a}}$ \\
\hline Preoperative neutrophil, $10^{9} / 1$ & $2.787 \pm 0.876$ & $4.417 \pm 2.875$ & $5.728 \pm 2.683$ & $<0.001$ \\
\hline Preoperative lymphocyte, $10^{9} / 1$ & $1.652 \pm 0.561$ & $1.319 \pm 0.499$ & $0.933 \pm 0.219$ & $0.012^{\mathrm{b}}$ \\
\hline Albumin, g/dl & $43.634 \pm 9.393$ & $39.685 \pm 2.757$ & $37.225 \pm 4.322$ & 0.144 \\
\hline Postoperative WBC, $10^{9} / 1$ & $5.603 \pm 1.870$ & $7.152 \pm 2.498$ & $7.218 \pm 2.981$ & $0.029^{c}$ \\
\hline Postoperative neutrophil, $10^{9} / 1$ & $3.427 \pm 1.354$ & $4.882 \pm 1.621$ & $5.695 \pm 2.300$ & $<0.001^{\mathrm{a}}$ \\
\hline Postoperative lymphocyte, $10^{9} / 1$ & $1.474 \pm 0.566$ & $1.254 \pm 0.918$ & $0.803 \pm 0.387$ & 0.094 \\
\hline Cirrhosis, yes & $24(45.3)$ & $7(53.8)$ & $2(50)$ & 0.852 \\
\hline Tumor size, $\mathrm{cm}$ & $5.111 \pm 3.622$ & $6.331 \pm 3.618$ & $10.850 \pm 5.555$ & $0.013^{\mathrm{d}}$ \\
\hline Tumor number, multiple & $10(18.86)$ & $0(0)$ & $2(50)$ & 0.027 \\
\hline Differentiation, poor & $5(9.43)$ & $1(7.69)$ & $2(50)$ & 0.044 \\
\hline Biliary leakage & $2(3.77)$ & $0(0)$ & $0(0)$ & 0.568 \\
\hline Pleural effusion & $42(79.24)$ & $10(76.9)$ & $4(100)$ & 0.391 \\
\hline Pneumonia & $3(5.66)$ & $0(0)$ & $0(0)$ & 0.425 \\
\hline Perihepatic effusion & $1(1.88)$ & $0(0)$ & $0(0)$ & 0.755 \\
\hline
\end{tabular}

${ }^{a}$ Data indicate the value $=0$ group vs. the value $=1$ and 2 groups; ${ }^{b}$ data indicate the value $=0$ group vs. the value $=2$ group; ${ }^{\mathrm{d}}$ ata indicate the value $=0$ group vs. the value $=1$ group; ${ }^{\mathrm{d}}$ data indicate the value $=2$ group vs. the other 2 groups. AFP, alpha-fetoprotein; HBsAg, hepatitis $B$ surface antigen; NLR, neutrophil-lymphocyte ratio; WBC, white blood cells.

classified as having a preoperative NLR plus postoperative NLR score of 1 and 2, respectively. Patients in the preoperative NLR plus postoperative NLR=2 group had a relatively larger tumor size than those in the preoperative NLR plus postoperative $\mathrm{NLR}=1 / 0$ group $(\mathrm{P}=0.013)$. Patients in the preoperative NLR plus postoperative NLR $=0$ group had relatively lower preoperative white blood cell $(\mathrm{P}=0.004)$ and postoperative neutrophil counts than those in the preoperative NLR plus postoperative $\mathrm{NLR}=2$ group $(\mathrm{P}<0.001)$. The preoperative lymphocytes were significantly lower in patients in the preoperative NLR plus postoperative $\mathrm{NLR}=2$ group than in those in the preoperative NLR plus postoperative $\mathrm{NLR}=0$ group $(\mathrm{P}=0.012)$. An increased preoperative NLR plus postoperative NLR score was associated with multiple tumors and poor differentiation. The demographic and clinicopathological features of the patients according to the preoperative NLR plus postoperative NLR score are shown in Table III.

\section{Discussion}

A high NLR reflects a decreased lymphocyte count and a relatively increased neutrophil count. Generally, neutrophils promote HCC progression and drug resistance (28), while lymphocytes may delay HCC progression (29). The mechanism by which inflammation promotes the development, progression and invasion of malignant tumors is complicated. The NLR may reflect the preoperative and postoperative immune responses. Morizawa et al (30) suggested that a high NLR was correlated with increased IL- 6 and IL- 8 and regulatory T cell expression. IL-6 prevents DNA-damage-induced hepatocyte apoptosis and enhances tumor proliferation, and IL-6 trans-signaling directly induces endothelial cell proliferation to promote tumor angiogenesis in a mouse HCC model (31). Cools-Lartigue et al (32) indicated that neutrophil extracellular trap deposition resulted in increased tumor cell adhesion to the hepatic microvasculature in vivo. Tumor cell trapping within neutrophil extracellular traps was associated with increased micrometastases (32).

Recently, numerous studies have indicated that an increased preoperative NLR is a reliable and economical independent prognostic indicator in different malignancies (2-8), and an increasing amount of research has focused on the effect of the postoperative inflammatory state on the prognosis of cancer (3,13-20,33). These studies have used traditional statistical methods, including ROC curves, to obtain optimal cut-offs (2-8,13-20,33). However, survival analysis is different from diagnostic experiments. The ROC curve may not be optimal in assessing models that stratify individuals into risk categories (34). Maximally selected rank statistics have been proven to be more effective for determining the cut-offs of continuous variables in survival analyses (26). Therefore, the present study used maximally selected rank statistics instead of ROC curves to obtain the best cut-offs. It is worth noting that certain studies have defined the time to obtain 

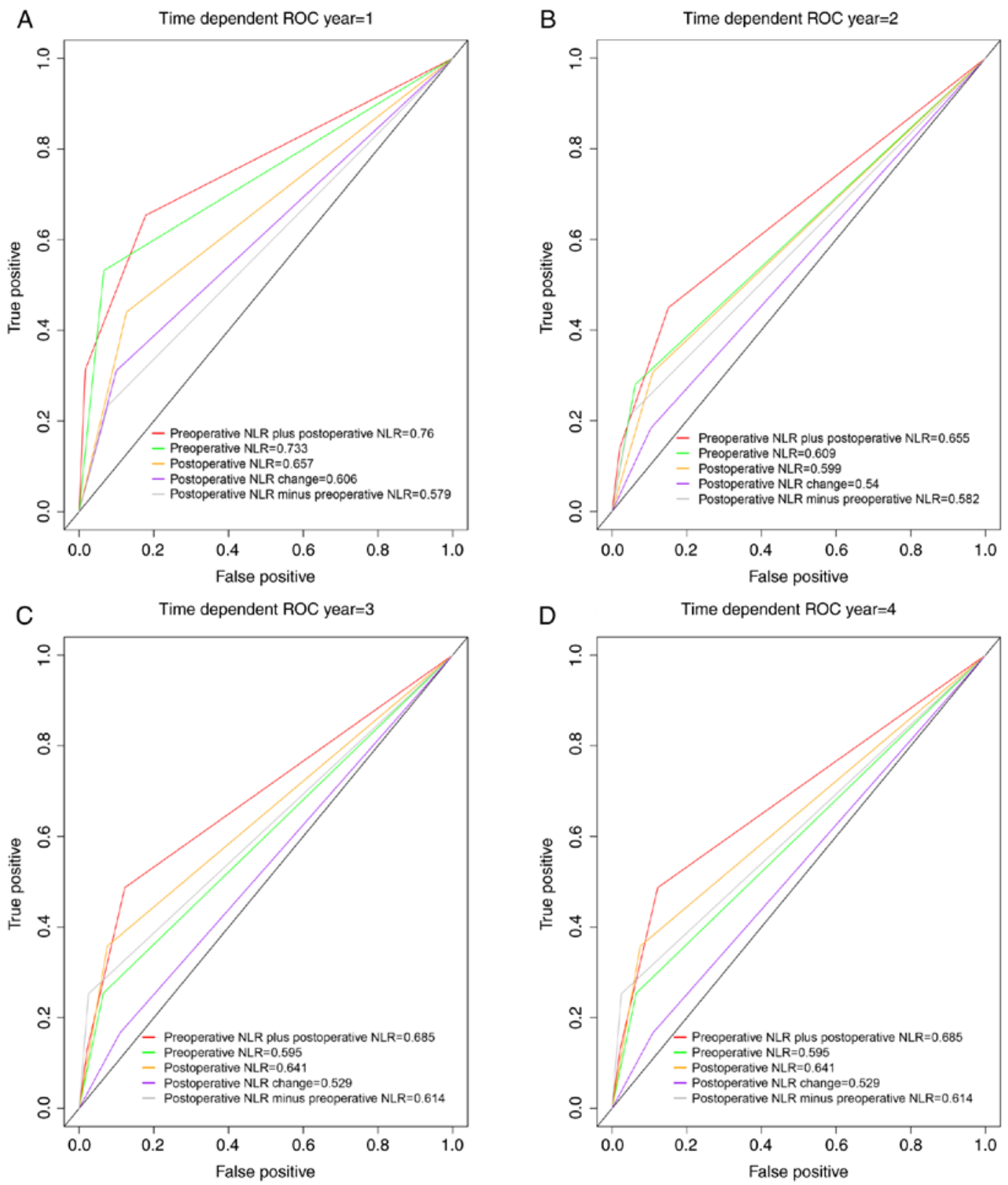

Figure 2. Comparison of the area under the time-dependent receiver operating characteristic curve for overall survival prediction. Comparisons among the inflammation indicators at (A) 1 year, (B) 2 years, (C) 3 years, and (D) 4 years in patients undergoing partial hepatectomy for hepatocellular carcinoma. ROC, receiver-operating characteristic; NLR, neutrophil-lymphocyte ratio.

postoperative NLR as 1 month after surgery, but there were no infections and complications in the exclusion criteria $(11,20)$. However, complications and infections may affect postoperative NLR (35). Certain studies have obtained postoperative NLR at least 1 month or 3-6 months after surgery $(18,19)$. The time interval for obtaining postoperative NLR was long, and the tumor may have progressed or recurred. Therefore, postoperative NLR at discharge was used in the present study. There are differences in the discharge standards for different hospitals. Patients are discharged only after they recover well (no infections and no unresolved complications) in our hospital. Therefore, the timing of obtaining the indicators in the present study may be relatively appropriate.

Although HCC has a different background to other solid tumors, and HCC is often based on chronic inflammation of the liver. To the best of our knowledge, no previous studies have compared the preoperative NLR of HCC with that of other solid tumors. However, consistent with HCC, the meta-analysis showed that patients with solid tumors in the high-NLR group had a poorer prognosis $(36,37)$. Zhou et al (38) found that their colorectal cancer group had a higher leukocyte count, neutrophil ratio and NLR than their adenomatous polyp group and healthy control group. This indicated that patients with a high tumor burden have a higher NLR. Miyatani et al (15) suggested that the preoperative NLR was significantly correlated with tumor burden (tumor size, tumor depth, lymphatic invasion and venous invasion), while there was no correlation between the postoperative NLR and the various clinicopathological variables. Shibutani et al (12) also found that the postoperative NLR was not associated with preoperative clinicopathological variables. The results of the present study are consistent with those of previous studies; the preoperative 
NLR was associated with tumor burden (tumor size) and preoperative inflammatory markers (preoperative white blood cell, neutrophil and lymphocyte counts), but there was no correlation between the preoperative NLR and postoperative inflammatory factors. Similarly, the postoperative NLR was associated with postoperative inflammatory markers, and the postoperative NLR was not associated with preoperative tumor burden or inflammatory markers. A possible reason for this is that the preoperative NLR represents the preoperative inflammatory status and high tumor burden prior to surgery, and the postoperative NLR represents the postoperative inflammatory status and potential tumor burden (for example, micrometastases). Peng et al (16) reported that the balance between the inflammatory response and the immune response may change following the surgical removal of HCC tumors. It is certain that the tumor burden following surgery is lower than that prior to surgery, but the association between postoperative inflammatory status and preoperative inflammatory status remains unclear. The immune status prior to surgery differs from that following surgery, and the optimal cut-offs for the preoperative NLR and the postoperative NLR were different. The present study indicated that using the postoperative NLR in the context of the preoperative NLR as a baseline to predict prognosis may not be very accurate. The prognostic effects of the preoperative NLR and postoperative NLR should be considered separately.

Recent studies have combined the preoperative NLR and postoperative NLR to study the prognostic impact on malignant tumors. To the best of our knowledge, the present study was the first to discuss the role of the preoperative NLR, postoperative NLR, and their different combinations in determining the prognosis of HCC. Similar to previous studies $(2,3,11,12)$, an increased preoperative NLR and an increased postoperative NLR were prognostic factors in the study; the difference is that the results of the present study suggested that an increased preoperative NLR and an increased postoperative NLR were not independent prognostic factors. However, the preoperative NLR plus postoperative NLR was an independent prognostic factor for OS in patients with HCC. Certain studies have divided the postoperative NLR by the preoperative NLR or used the post NLR minus the preoperative NLR to define novel prognostic factors $(3,13,14,16-20)$. Certain studies have hypothesized that an increase in the postoperative NLR relative to the preoperative NLR following treatment indicates that the balance has been tipped in favor of a pro-tumor inflammatory response $(3,16-20)$. However, Lalani et al (13) found that a relative NLR change of $\geq 25 \%$ from baseline to 6 weeks after PD-1/PD-L1 immune checkpoint blockade therapy was an independent prognostic factor for OS. Unlike previous studies (13), the present study found that the postoperative NLR cut-off was -0.25 , instead of -0.25 and 0.25 as defined using traditional statistical methods. The present study demonstrated a poor prognosis for a postoperative NLR change reduction of $<25 \%$, but the prognostic difference between the two groups was not statistically significant. The optimal cut-off for the postoperative NLR minus preoperative NLR in this study was 3.42 , rather than $0(3,16,19,20), 0.037$ (17) or 1.5 (18), as obtained using traditional statistical methods. The results of the present study suggested that the postoperative NLR change and postoperative NLR minus preoperative NLR were not independent prognostic factors for OS. Although the postoperative NLR minus preoperative NLR was an independent prognostic factor for HCC in previous studies, no significant differences were identified between the two groups in terms of commonly used clinicopathological features $(3,16)$. Unfortunately, no clinical pathology data were compared in one previous postoperative NLR change prognostic study (13). As the mechanisms causing a high NLR prior to and following surgery are not consistent, the relative changes (postoperative NLR change and postoperative NLR minus preoperative NLR) in the postoperative NLR may not reflect the true immune status following surgery. This may be the reason why there was no significant difference in pathological parameters between the postoperative NLR minus preoperative NLR groups $(3,16)$.

In the present study, the postoperative NLR plus preoperative NLR was first identified as an improved prognostic factor for HCC following hepatectomy compared with the preoperative NLR, postoperative NLR, postoperative NLR change, and postoperative NLR minus preoperative NLR groups according to td-ROC curves. The postoperative NLR plus preoperative NLR groups had the largest td-AUC in the first to fourth years. This suggests that the combination of the preoperative NLR and the postoperative NLR with the postoperative NLR plus preoperative NLR is more effective than the postoperative NLR change or the postoperative NLR minus preoperative NLR in predicting the prognosis of patients with HCC. The preoperative NLR plus postoperative NLR was also more useful for predicting the prognosis of patients with HCC than either the preoperative NLR or the postoperative NLR. Multivariate analysis identified the preoperative NLR plus postoperative NLR as an independent prognostic risk factor. Miyatani et al (15) also reported that a high preoperative and a high postoperative NLR were independent prognostic indicators for gastric cancer. In the present study, patients in the preoperative NLR plus postoperative NLR=2 group had larger tumor sizes than those in the preoperative NLR plus postoperative NLR $=1 / 0$ group. An increased preoperative NLR plus postoperative NLR score was associated with multiple tumors and poor differentiation. Several studies have indicated that multiple tumor numbers, larger tumor sizes and poor differentiation are predictors of a poor outcome (39-41). Similarly, the results of the present study suggested that an increased preoperative NLR plus postoperative NLR was associated with preoperative and postoperative inflammatory indicators. Taken together, the results of the present study suggested that the preoperative NLR plus postoperative NLR more comprehensively reflects the preoperative and postoperative tumor burden and inflammatory or immune status than the other parameters; therefore, the preoperative NLR plus postoperative NLR may be a more reliable prognostic factor. The preoperative NLR plus postoperative NLR considers the two preoperative and postoperative treatment phases, and thus, the combined indicators may more accurately predict the prognosis, compared with individual indicators. Lalani et al (13) found that a postoperative NLR decrease $\geq 25 \%$ from baseline to 6 weeks was associated with an improved OS, but only a postoperative NLR increase by $\geq 25 \%$ was an independent prognostic factor for OS following PD-1/PD-L1 immune checkpoint blockade therapy. We hypothesized that the postoperative NLR may be somewhat associated with the immunotherapy response. 
The present study has certain limitations. To begin with, the present study is a retrospective and single-center study with possible unmeasurable selection biases. Furthermore, the small sample size of this study may limit the assessment of the prognostic value of different influencing factors. Additionally, heterogeneity of the patient's liver disease background, including patients with HBV or HCV-related HCC, may affect the NLR cut-off. Finally, the preoperative NLR and postoperative NLR require further investigation in immunotherapy studies, and it is necessary to identify which neutrophil and lymphocyte subsets have prognostic utility.

Compared with conventional prognostic factors, including preoperative NLR, postoperative NLR, preoperative NLR plus postoperative NLR, change in postoperative NLR, and postoperative NLR minus preoperative NLR, the preoperative NLR plus postoperative NLR, considering both the preoperative and postoperative treatment phase, may be a more effective prognostic factor for patients with HCC and requires further investigation in the future.

\section{Acknowledgements}

The authors would like to thank Dr Zhaoyang Liu (Statistical Consultant Center, Tsinghua University) for providing statistical assistance.

\section{Funding}

The present study was supported by the National Science \& Technology Major Project (grant no. 2017ZX10203205), National Natural Science Foundation of China (grant no. 81930119), and the Medical-Engineering Project of Tsinghua University School of Software (grant no. MESR201912-3).

\section{Availability of data and materials}

The datasets used and/or analyzed during the present study are available from the corresponding author on reasonable request.

\section{Authors' contributions}

MW and SY are responsible for the study concept and design, methodology, software, data collection and analyses, writing the initial draft of the manuscript and critically revising the manuscript. XF is responsible for the data analysis, methodology and critically revising the manuscript. FY and XL are responsible for the data collection. JD is responsible for the study concept and design, methodology, critical revision of the manuscript, and project administration and supervision. All authors read and approved the final manuscript.

\section{Ethics approval and consent to participate}

The present study was approved by the Ethics Committee of Beijing Tsinghua Chang Gung Hospital (Beijing, China).

\section{Patient consent for publication}

Not applicable.

\section{Competing interests}

The authors declare that they have no competing interests.

\section{References}

1. Yang JD and Roberts LR: Hepatocellular carcinoma: A global view. Nat Rev Gastroenterol Hepatol 7: 448-458, 2010

2. Oh BS, Jang JW, Kwon JH, You CR, Chung KW, Kay CS, Jung HS and Lee S: Prognostic value of C-reactive protein and neutrophil-to-lymphocyte ratio in patients with hepatocellular carcinoma. BMC Cancer 13: 78, 2013.

3. Dan J, Zhang Y, Peng Z, Huang J, Gao H, Xu L and Chen M: Postoperative neutrophil-to-lymphocyte ratio change predicts survival of patients with small hepatocellular carcinoma undergoing radiofrequency ablation. PLoS One 8: e58184, 2013.

4. Lopes M, Carvalho B, Vaz R and Linhares P: Influence of neutrophil-lymphocyte ratio in prognosis of glioblastoma multiforme. J Neurooncol 136: 173-180, 2018.

5. Luo G, Liu C, Cheng H, Jin K, Guo M, Lu Y, Long J, Xu J, Ni Q, Chen J and Yu X: Neutrophil-lymphocyte ratio predicts survival in pancreatic neuroendocrine tumors. Oncol Lett 13: 2454-2458, 2017.

6. Jin H, Sun J, Zhu K, Liu X, Zhang Q, Shen Q, Gao Y and Yu J: The prognostic value of neutrophil-lymphocyte ratio is superior to derived neutrophil-lymphocyte ratio in advanced gastric cancer treated with preoperative chemotherapy and sequential R0 resection: A 5-year follow-up. Onco Targets Ther 10: 2655-2664, 2017.

7. Komura N, Mabuchi S, Yokoi E, Kozasa K, Kuroda H, Sasano T, Matsumoto Y and Kimura T: Comparison of clinical utility between neutrophil count and neutrophil-lymphocyte ratio in patients with ovarian cancer: A single institutional experience and a literature review. Int J Clin Oncol 23: 104-113, 2018.

8. Sun Z, Ju Y, Han F, Sun X and Wang F: Clinical implications of pretreatment inflammatory biomarkers as independent prognostic indicators in prostate cancer. J Clin Lab Anal 32: e22277, 2018.

9. Ethier JL, Desautels D, Templeton A, Shah PS and Amir E: Prognostic role of neutrophil-to-lymphocyte ratio in breast cancer: A systematic review and meta-analysis. Breast Cancer Res 19: 2, 2017.

10. Grivennikov SI, Greten FR and Karin M: Immunity, inflammation, and cancer. Cell 140: 883-899, 2010.

11. Lee SK, Choi MY, Bae SY, Lee JH, Lee HC, Kil WH, Lee JE, Kim SW and Nam SJ: Immediate postoperative inflammation is an important prognostic factor in breast cancer. Oncology 88: 337-344, 2015.

12. Shibutani M, Maeda K, Nagahara H, Ohtani H, Iseki Y, Ikeya T, Sugano K and Hirakawa K: The prognostic significance of a postoperative systemic inflammatory response in patients with colorectal cancer. World J Surg Oncol 13: 194, 2015.

13. Lalani AA, Xie W, Martini DJ, Steinharter JA, Norton CK, Krajewski KM, Duquette A, Bossé D, Bellmunt J, Van Allen EM, et al: Change in Neutrophil-to-lymphocyte ratio (NLR) in response to immune checkpoint blockade for metastatic renal cell carcinoma. J Immunother Cancer 6: 5, 2018.

14. Li M, Spakowicz D, Burkart J, Patel S, Husain M, He K, Bertino EM, Shields PG, Carbone DP, Verschraegen CF, et al: Change in neutrophil to lymphocyte ratio during immunotherapy treatment is a non-linear predictor of patient outcomes in advanced cancers. J Cancer Res Clin Oncol 145: 2541-2546, 2019.

15. Miyatani K, Saito H, Kono Y, Murakami Y, Kuroda H, Matsunaga T, Fukumoto Y, Osaki T, Ashida K and Fujiwara Y: Combined analysis of the pre- and postoperative neutrophil-lymphocyte ratio predicts the outcomes of patients with gastric cancer. Surg Today 48: 300-307, 2018.

16. Peng W, Li C, Wen TF, Yan LN, Li B, Wang WT, Yang JY and $\mathrm{Xu}$ MQ: Neutrophil to lymphocyte ratio changes predict small hepatocellular carcinoma survival. J Surg Res 192: 402-408, 2014.

17. Guo D, Han A, Jing W, Chen D, Jin F, Li M, Kong L and Yu J: Preoperative to postoperative change in neutrophil-to-lymphocyte ratio predict survival in colorectal cancer patients. Future Oncol 14: 1187-1196, 2018.

18. Jin F, Han A, Shi F, Kong L and Yu J: The postoperative neutrophil-to-lymphocyte ratio and changes in this ratio predict survival after the complete resection of stage I non-small cell lung cancer. OncoTargets Ther 9: 6529-6537, 2016. 
19. Min KW, Kwon MJ, Kim DH, Son BK, Kim EK, Oh YH and Wi YC: Persistent elevation of postoperative neutrophil-to-lymphocyte ratio: A better predictor of survival in gastric cancer than elevated preoperative neutrophil-to-lymphocyte ratio. Sci Rep 7: 13967, 2017.

20. Zhou ZQ, Pang S, Yu XC, Xue Q, Jiang HY, Liang XJ and Liu L: Predictive values of postoperative and dynamic changes of inflammation indexes in survival of patients with resected colorectal cancer. Curr Med Sci 38: 798-808, 2018.

21. Nishihara K, Suekane S, Ueda K, Nakiri M, Matsuo M and Igawa T: High postoperative neutrophil-to-lymphocyte ratio as a poorprognostic marker in patients with upper tract urothelial carcinoma. Oncol Lett 17: 5241-5250, 2019.

22. El-Serag HB and Rudolph KL: Hepatocellular carcinoma: Epidemiology and molecular carcinogenesis. Gastroenterology 132: 2557-2576, 2007.

23. Martins-Filho SN, Paiva C, Azevedo RS and Alves VAF: Histological grading of hepatocellular carcinoma-a systematic review of literature. Front Med (Lausanne) 4: 193, 2017.

24. Huang SS, Xie DM, Cai YJ, Wu JM, Chen RC, Wang XD, Song M, Zheng MH, Wang YQ, Lin Z and Shi KQ: C-reactive protein-to-albumin ratio is a predictor of hepatitis $B$ virus related decompensated cirrhosis: Time-dependent receiver operating characteristics and decision curve analysis. Eur J Gastroenterol Hepatol 29: 472-480, 2017.

25. Kamarudin AN, Cox $\mathrm{T}$ and Kolamunnage-Dona R: Timedependent ROC curve analysis in medical research: Current methods and applications. BMC Med Res Methodol 17: 53, 2017.

26. Hothorn T and Zeileis A: Generalized maximally selected statistics. Biometrics 64: 1263-1269, 2008.

27. Martin D, Rodel F, Balermpas P, Winkelmann R, Fokas E and Rodel C: C-reactive protein-to-albumin ratio as prognostic marker for anal squamous cell carcinoma treated with chemoradiotherapy. Front Oncol 9: 1200, 2019.

28. Zhou SL, Zhou ZJ, Hu ZQ, Huang XW, Wang Z, Chen EB, Fan J, Cao Y, Dai Z and Zhou J: Tumor-associated neutrophils recruit macrophages and T-regulatory cells to promote progression of hepatocellular carcinoma and resistance to sorafenib. Gastroenterology 150: 1646-1658 e1617, 2016.

29. Nakagawa S, Umezaki N, Yamao T, Kaida T, Okabe H, Mima K, Imai K, Hashimoto D, Yamashita YI, Ishiko T, et al: Survival impact of lymphocyte infiltration into the tumor of hepatocellular carcinoma in hepatitis B virus-positive or non-B non-C patients who underwent curative resection. Hepatol Res 48: E126-E132, 2018.

30. Morizawa Y, Miyake M, Shimada K, Hori S, Tatsumi Y, Nakai Y, Onishi S, Tanaka N, Konishi N and Fujimoto K: Correlation of immune cells and cytokines in the tumor microenvironment with elevated neutrophil-to-lymphocyte ratio in blood: An analysis of muscle-invasive bladder cancer. Cancer Invest 36: 395-405, 2018

31. Bergmann J, Muller M, Baumann N, Reichert M, Heneweer C, Bolik J, Lücke K, Gruber S, Carambia A, Boretius S, et al: IL-6 trans-signaling is essential for the development of hepatocellular carcinoma in mice. Hepatology 65: 89-103, 2017.
32. Cools-Lartigue J, Spicer J, McDonald B, Gowing S, Chow S, Giannias B, Bourdeau F, Kubes P and Ferri L: Neutrophil extracellular traps sequester circulating tumor cells and promote metastasis. J Clin Invest 123: 3446-3458, 2013.

33. Li C, Wen TF, Yan LN, Li B, Wang WT, Yang JY and Xu MQ: Postoperative neutrophil-to-lymphocyte ratio plus platelet-to-lymphocyte ratio predicts the outcomes of hepatocellular carcinoma. J Surg Res 198: 73-79, 2015.

34. Cook NR: Use and misuse of the receiver operating characteristic curve in risk prediction. Circulation 115: 928-935, 2007.

35. Medina Fernandez FJ, Munoz-Casares FC, Arjona-Sanchez A, Casado-Adam A, Gómez-Luque I, Garcilazo Arismendi DJ, Thoelecke H, Rufián Peña S and Briceño Delgado J: Postoperative time course and utility of inflammatory markers in patients with ovarian peritoneal carcinomatosis treated with neoadjuvant chemotherapy, cytoreductive surgery, and HIPEC. Ann Surg Oncol 22: 1332-1340, 2015.

36. Mei Z, Shi L, Wang B, Yang J, Xiao Z, Du P, Wang Q and Yang W: Prognostic role of pretreatment blood neutrophil-to-lymphocyte ratio in advanced cancer survivors: A systematic review and meta-analysis of 66 cohort studies. Cancer Treat Rev 58: 1-13, 2017.

37. Templeton AJ, McNamara MG, Seruga B, Vera-Badillo FE, Aneja P, Ocaña A, Leibowitz-Amit R, Sonpavde G, Knox JJ, Tran B, et al: Prognostic role of neutrophil-to-lymphocyte ratio in solid tumors: A systematic review and meta-analysis. J Natl Cancer Inst 106: dju124, 2014.

38. Zhou WW, Chu YP and An GY: Significant difference of neutrophil-lymphocyte ratio between colorectal cancer, adenomatous polyp and healthy people. Eur Rev Med Pharmacol Sci 21: 5386-5391, 2017.

39. Hwang S, Lee YJ, Kim KH, Ahn CS, Moon DB, Ha TY, Song GW, Jung DH and Lee SG: The impact of tumor size on long-term survival outcomes after resection of solitary hepatocellular carcinoma: Single-institution experience with 2558 patients. J Gastrointest Surg 19: 1281-1290, 2015.

40. Wang X, Wang $Z$ and Wu L: Combined measurements of tumor number and size helps estimate the outcome of resection of Barcelona clinic liver cancer stage B hepatocellular carcinoma. BMC Surg 16: 22, 2016.

41. Zhang TT, Zhao XQ, Liu Z, Mao ZY and Bai L: Factors affecting the recurrence and survival of hepatocellular carcinoma after hepatectomy: A retrospective study of 601 Chinese patients. Clin Transl Oncol 18: 831-840, 2016.

This work is licensed under a Creative Commons Attribution-NonCommercial-NoDerivatives 4.0 International (CC BY-NC-ND 4.0) License. 